\title{
Letter from the Guest Editors
}

This issue of the JBCS is dedicated to computer networks and distributed systems. The papers have been selected from those presented during SBRC'01 that was held in Florianópolis, Island of Santa Catarina. The Brazilian Symposium on Computer Networks (SBRC) is an annual event promoted by the Brazilian Computer Society (SBC) and by the National Laboratory of Computer Networks (LARC).

The Technical Program Committee (TPC) selected 56 papers out of 204 submissions received from Brasil, USA, France, Portugal, Korea, England, and Netherlands. The selected papers covered research topics and applications in High-Speed Networks, Middleware, Multicomputers, Distributed Processing, and Mobile Computing.

Among the high quality technical papers accepted by the Symposium, the TPC selected the 10 best papers and invited authors to submit an extended version of their contribution to the Journal of the Brazilian Computer Society. These new versions were then rated by TPC members, and finally were thoroughly reviewed by the JBCS Editor, who choose 7 papers for publication in this special issue.

The first three papers are related to multicast communications. The first one proposes a novel routing protocol, which allows a flexible deployment of the multicast service. The second paper thoroughly analyses different multicast protocols that modify Deering's model, identifying their weaknesses and strengths. The third paper proposes two mechanisms to improve the performance of multi-layered video distribution. The fourth paper presents an environment tool designed for the performance analysis of traffic enginneering mechanisms, such as CAC algorithms. The fifth paper proposes a framework for the creation of mobile transport protocols that simultaneously use multiple link layers. The sixth paper focuses on mobile agents, and proposes a mechanism for the coordination and synchronization of information on agent location and addressing. Finally, the last paper is on network management: the authors propose a graphical/textual language that allows network managers to program the monitoring agents inside the network.

We would like to thank all the members of the Technical Program Committee for their invaluable support in the reviewing process. We also want to thank the members of the TPC that cooperated in the final selection of the seven papers, selection made through the blind review method. Finally, we would like to give special thanks to the editors of the JBCS, Marco Antonio Casanova and Paulo Cesar Masiero.

Otto Carlos Muniz Bandeira Duarte and Julius Leite

Guest Editors, Special Issue on Computer Networks and Distributed Systems 